\title{
A Neural Computational Intelligence Method Based on Legendre Polynomials for Fuzzy Fractional Order Differential Equation
}

\author{
N. A. KHAN, A. SHAIKH, M. A. ZAHOOR RAJA AND S. KHAN
}

\begin{abstract}
In this article, Legendre simulated annealing, neural network (LSANN) is designed for fuzzy fractional order differential equations, which is employed on fractional fuzzy initial value problem (FFIVP) with triangular condition. Here, Legendre polynomials are used to modify the structure of neural networks with a Taylor series approximation of the tangent hyperbolic as activation function while the network adaptive coefficients are trained in the procedure of simulated annealing to optimize the residual error. The computational results are depicted in terms of numerical values to compare them with previous results.
\end{abstract}

Mathematics Subject Classification 2010: 34A07, 42C10, 62M45

Additional Key Words and Phrases: Fuzzy; neural network; simulated annealing; Legendre polynomials

\section{INTRODUCTION}

In 1695, Leibniz raised the problem of fractional derivative, which became the base of fractional calculus; fractional derivative and fractional integral operators, which is an extension of the familiar derivative and integral operator to real values. Interest in fractional calculus has been inspired by the applications that it finds in the modeling of physical phenomena such as rheology, viscoelasticity, electrochemistry, diffusion processes, earthquakes modeling, fluid dynamics, traffic model, etc. The recently influential works on the subject of fractional order is done by many researchers such as Li et al. [1] analyzed the nonlinear system of fractional order by using phase portraits, Poincare maps, time domain waveforms, and bifurcation diagrams, Pourdehi et al. [2] investigated a modified fuzzy Kalman-type filter for a fractional order system with finite-step auto-correlated process noise, Boulkroune et al. [3] proposed a novel fuzzy adaptive controller for achieving an appropriate 
generalized projective synchronization (GPS) of two incommensurate fractionalorder chaotic systems, Ji et al. [4] focused on the uncertain fractional order systems (FOS), Zheng [5] studied the approach to stabilize the fractional order chaotic system by utilizing the prediction based feedback controls and Takagi-Sugeno fuzzy models. All the above fractional models have solutions that are continuous fractions, but they are non-differentiable such as Weierstrass type functions. Ordinary differential models cannot deal with the models of such complex phenomena consequently modeling in terms of fractional differential equations filled this gap. It is difficult to find the exact solution of fractional differential equations, so different researchers used different approaches and techniques like Improved Euler method [6], which is an extension of classical Euler's method, modified fractional Euler method (MFEM) [7], modified Adams-Bashforth-Moulton method (MABMM) [8], linear matrix inequalities (LMIs) control toolbox [9], Chattering-free robust method [10], Mellin transformation, differential transformation method (DTM), and Laplace transformation [11].

Nowadays, real world problems are complex and this complexity generally arises from the vagueness of the colloquial language. For handling such complexities that do not allow them to be studied by classical procedures based on probability theory and bivalent logic Zadeh introduced fuzzy logic in 1965 [2]. In the beginning, this theory appeared unsustainable, but further progress proved that Zadeh's intuitions were right, beyond all anticipations. Due to a number of applications in mathematical modeling, it got the attention of researchers to develop different methods to solve these models. Tapaswami et al. [12] gave the solution of heat conduction problem with uncertainty in which uncertainty is modeled by triangular convex normalized fuzzy set and the modeled problem is solved by the variational iteration method (VIM) with a double parametric form of fuzzy numbers. Ahmadian et al. [13] derived shifted Legendre operational matrix for solving fuzzy fractional order differential equations. Allahviranloo et al. [14] applied AdamsBashforth, Adams-Moulton, and predictor-corrector methods to solve fuzzy ordinary differential equations. Ahmad et al. [15] proposed numerical solution of 
fuzzy differential equations by fuzzification of classical Euler's method and optimization method. Khastan and Ivaz [16] employed Nyström method to solve fuzzy first order initial value problem. EIJaoui, et al. [17] used fuzzy Laplace transformation to solve first and second order fuzzy differential equation under comprehensive Hukuhara differentiability. Khan et al. [18, 19] investigated the fuzzy differential equations by modifying the homotopy perturbation method, improved Euler's method, and max-min improved Euler's method.

In recent years, artificial neural networks (ANN) become one of the most useful techniques to solve real world problems. This technique is based on neural network models. This method is flexible and can generally be applied to solve different type of linear and nonlinear singular or initial value problems. Unlike other numerical methods, this method gave a continuous approximate solution over the domain of integration. Moreover, other numerical methods like Runge-Kutta (RK) and predictor-corrector methods are iterative for which the step size should be fixed before initiating the computation. After obtaining the solution, if one needs to know the solution in between of any steps, the process needs to be repeated from primary stage. One can also use interpolation methods to get approximate solutions, but sometimes interpolation methods are error prone. The Artificial neural network gave relief from repetition of iterative methods and interpolations. Also, one may use it as a black box to get numerical results at any arbitrary point in the domain [20]-[21].

Meade and Fernandez [22]-[23] have calculated the linear ordinary differential equations by using a feed forward neural networks. Lagaris et al. [24] [25] have solved the ordinary and partial differential equations with initial/ boundary values and complex boundary by utilizing artificial neural networks (ANN). Parisi et al. [26] have investigated the powerful problem of chemical engineering (a non-steady state fixed bed solid-gas reactor). Malek and Beidokhti [27] have studied higher order differential equations using the hybrid technique based on optimization and neural network methods. 


\section{PRELIMINARIES}

In this section, the basic concepts of Caputo fractional derivative, fuzzy number and a Triangular fuzzy number are summarized. For detailed study of fuzzy theory, interested reader can read [28,29].

DEFINITION 2.1. The fractional derivative of $g(x)$ in Caputo sense of order $\alpha>0$ is defined as [30, 31].

$D_{*}^{\alpha} g(x)=\frac{1}{\Gamma(\eta-\alpha)} \int_{a}^{x} \frac{g^{(\eta)}(\beta)}{(x-\beta)^{\alpha+1-\eta}} d \beta, \quad \eta-1<\alpha<\eta$,

where $D_{*}^{\alpha}$ is the Caputo derivative operator $\alpha>0, x>a, \alpha, a$ and $x \in R$ The Caputo fractional derivative becomes zero when applied to a constant.

DEFINITION 2.2. Let $\mathfrak{R}$ denote the universe of discourse, then a function $\tilde{A}: \mathfrak{R} \rightarrow[0,1]$ defines a fuzzy set on $\mathfrak{R}$, known as membership function, which holds the following properties [32]

- $\tilde{A}$ is upper semi-continuous on $\mathfrak{R}$,

- $\quad \tilde{A}$ is fuzzy convex, i.e., $\tilde{A}(\xi x+(1-\xi) y) \geq \min \{\tilde{A}(x), \tilde{A}(y)\}$ for all $\forall$ $x, y \in \mathfrak{R}, \xi \in[0,1]$,

- $\quad \tilde{A}$ is normal, i.e. $\exists x_{0} \in \mathfrak{R}$ for which $\tilde{A}\left(x_{0}\right)=1$,

- $\operatorname{supp} \tilde{A}=\{x \in \mathfrak{R} \mid \tilde{A}(x)>0\}$ is the support of $\tilde{A}$, and its $\operatorname{closure} \operatorname{cl}(\operatorname{supp} \tilde{A})$ is compact.

The fuzzy set can be represented by $\tilde{A}=\left\{\left\langle x, u_{\tilde{A}}(x)\right\rangle: x \in \mathfrak{R}\right\}$, where $u_{\tilde{A}}(x)$ is called membership grade of $x, \forall x \in \mathfrak{R}$ each pair $\left\langle x, u_{\tilde{A}}(x)\right\rangle$ is called a singleton.

An equivalent parametric definition is also given in [21][22][23] as follows: 
DEFINITION 2.3. An arbitrary fuzzy number into parametric form is defined as [32] an ordered pair of function $(\underline{u}(r), \bar{u}(r)), 0<r<1$ in which $\underline{u}(r)$ and $\bar{u}(r)$ are correspondingly monotonically increasing and monotonically decreasing bounded, left continuous on $[0,1]$ and right continuous on 0 with the following property. $\underline{u}(r) \leq \bar{u}(r), 0 \leq r \leq 1$.

DEFINITION 2.4. The $r$-cut ( $r$ level or $r$ worthy) of a fuzzy set is a crisp set described as [33] ${ }^{r} \tilde{A}=\{x \mid \tilde{A}(x) \geq r\}, 0<r \leq 1, x \in \mathfrak{R}$.

While strong $r$-cut set can be demonstrated as ${ }^{r+} \tilde{A}=\{x \mid \tilde{A}(x)>r\}, 0<r \leq 1, x \in \mathfrak{R}$.

Let $r_{1}$ and $r_{2}$ are two different values of $r$-cut moreover $\tilde{A}$ and $\tilde{B}$ belong to fuzzy set then $r$-cut and strong $r$-cut possess following properties

- ${ }^{r+} \tilde{A} \subseteq{ }^{r} \tilde{A}$.

- $r_{1} \leq r_{2} \Rightarrow{ }^{r 1} \mathrm{~A} \supseteq^{r 2} A$ and ${ }^{r 1+} A \supseteq^{r 2+} A$;

- $\quad r(\tilde{A} \cap \tilde{B})={ }^{r} \tilde{A} \cap{ }^{r} \tilde{B}$ and ${ }^{r}(\tilde{A} \cup \tilde{B})={ }^{r} \tilde{A} \cup{ }^{r} \tilde{B}$

- $\quad{ }^{r+}(\tilde{A} \cap \tilde{B})={ }^{r+} \tilde{A} \cap^{r+} \tilde{B}$ and ${ }^{r+}(\tilde{A} \cup \tilde{B})={ }^{r+} \tilde{A} \cup{ }^{r+} \tilde{B}$

- $r(\overline{\tilde{A}})=^{(1-r)+(}(\overline{\tilde{A}})$

DEFINITION 2.5. Let $\tilde{u}$ and $\tilde{v}$ be two fuzzy numbers with $\tilde{u}_{A}$ and $\tilde{v}_{A}$ as their membership function, respectively, then their sum is defined as [34] $\tilde{u} \oplus \tilde{v}=\max _{x+y=z} \min \left(\tilde{u}_{A}(x), \tilde{v}_{A}(y)\right),(x, y, z) \in R^{3}$.

While, the product of two fuzzy numbers can be demonstrated as $\tilde{u} \otimes \tilde{v}$ $\tilde{u} \otimes \tilde{v}=\max _{x y=z} \min \left(\tilde{u}_{A}(x), \tilde{v}_{A}(y)\right),(x, y, z) \in R^{3}$. 
DEFINITION 2.6. A convex normalized set of fuzzy number of a real line that is described by an ordered triple is known as a triangular fuzzy number. Let the fuzzy number be $A=\left(a_{1}, a_{2}, a_{3}\right)$ such that $a_{1}<a_{2}<a_{3}$ then the membership function is interpreted as [6].

$$
u_{A}(x)=\left\{\begin{array}{l}
\frac{x-a_{1}}{a_{2}-a_{1}}, \text { if } a_{1} \leq x \leq a_{2} \\
\frac{a_{3}-x}{a_{3}-a_{2}}, \text { if } a_{2} \leq x \leq a_{3} \\
0, \quad \text { otherwise }
\end{array}\right.
$$

The $r$-level set of a triangular fuzzy number is given by

$$
{ }^{r} \tilde{A}=\left[a_{2}-(1-r)\left(a_{2}-a_{1}\right), a_{2}+(1-r)\left(a_{3}-a_{2}\right)\right]
$$

\section{LSANN FORMULATION FOR FRACTIONAL DIFFERENTIAL EQUATIONS}

In this section, the implementation of LSANN on a generalized form of fractional differential equation will be demonstrated that characterized the ordinary or partial differential equation [22].

$G\left(t, y(t), \nabla^{\alpha} y(t), \nabla^{\beta} y(t), \nabla^{m} y(t)\right)=0, \quad t \in D \subseteq R^{n}, \quad \alpha, \beta>m$,

subject to

$y(a)=b$,

where $\mathrm{G}$ demonstrated the structure of fractional differential equation, $\nabla$ is a differential operator, $y(t)$ is the solution to be calculated and $\tilde{D}$ is the discretized domain over a finite set of points. Let $y_{t}(t, w)$ symbolizes the trial solution with modifiable network adaptive coefficients (weights) and formulated according to [24]. 
$y_{t}(t, w)=\delta(t)+F(t, N(t, w))$

where, $\delta(t)$ fulfills only initial/boundary conditions, the output of LSANN is $N(t, w)$ and $w$ are the weights of LSANN then Eq. (2) in terms of trial solution and discretized array of input points may be formulated as,

$G\left(t, y_{t}(t, w), \nabla^{\beta} y_{t}(t, w), \nabla^{\alpha} y_{t}(t, w) \nabla^{m} y_{t}(t, w)\right)=0, \quad t \in \tilde{D}$

While, $N(t, w)$ can be calculated from the network as follows. Here, the first three terms of the Taylor series of Tangent hyperbolic function are used as activation function.

$$
N(t, w)=\phi-\frac{\phi^{3}}{3}+\frac{2 \phi^{5}}{15}
$$

and

$\phi=\sum_{j=1}^{n} w_{j} L_{j-1}(t)$

While, $L_{j-1}$ are Legendre polynomials with following recursive formula,

$$
\begin{aligned}
& L_{j+1}=\frac{(2 j+1)}{(j+1)} x L_{j}(x)-\frac{j}{(j+1)} L_{j-1}(x), l>1 \\
& L_{0}(x)=1 \text { and } L_{l}(x)=x
\end{aligned}
$$

The structure of Legendre Neural network is further described in Fig. 1. 


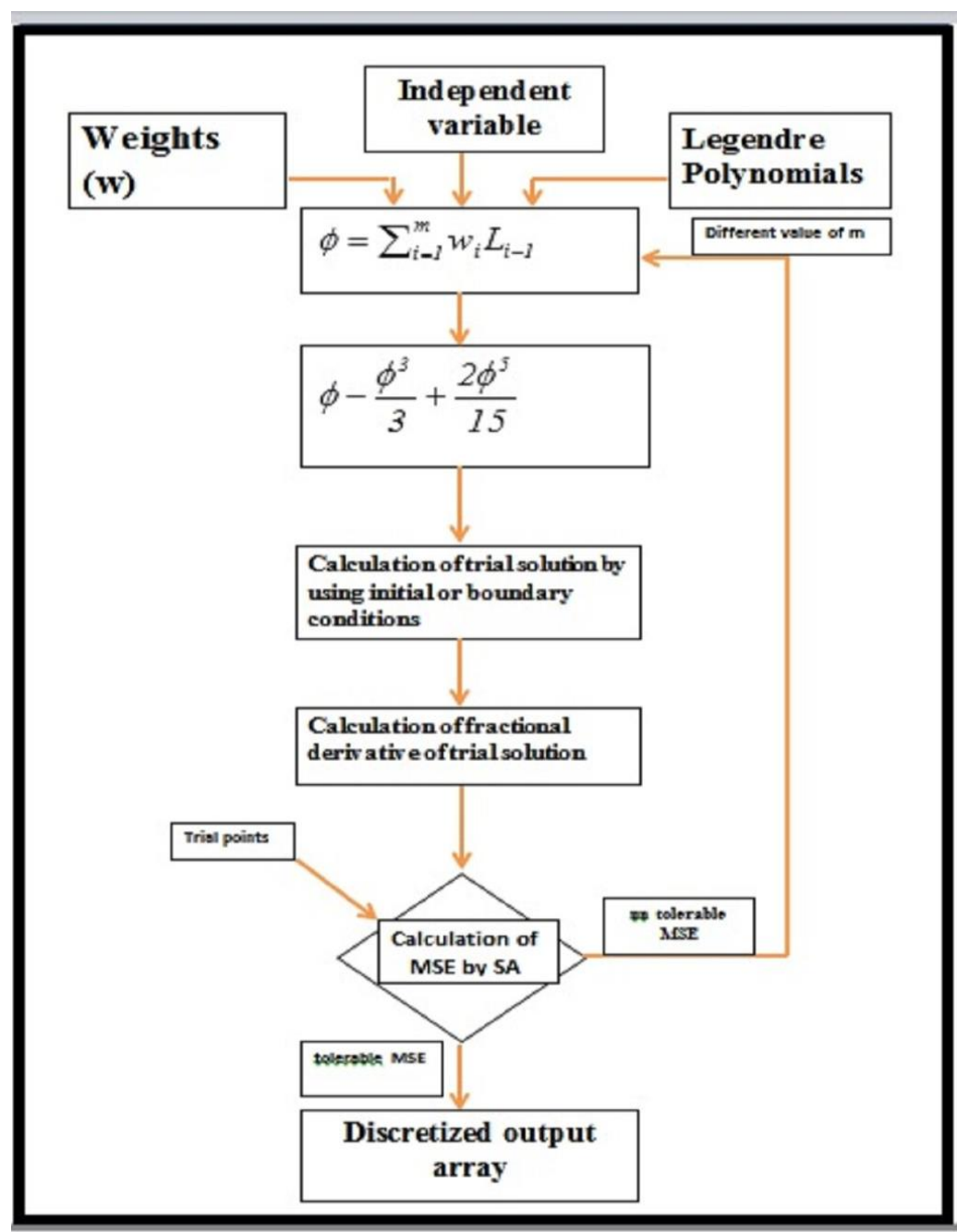

Fig. 1. Structure of Legendre Neural Network.

The mean square error (MSE) for Eq. (3) is given by

$$
\operatorname{MSE}\left(w_{j}\right)=\sum_{i=1}^{H} \frac{1}{H}\left\{\nabla^{m} y_{t}\left(t_{i}, w_{j}\right)-f\left(t_{i}, y_{t}\left(t_{i}, w_{j}\right), \nabla^{\alpha} y_{t}\left(t_{i}, w_{j}\right), \nabla^{\beta} y_{t}\left(t_{i}, w_{j}\right)\right)\right\}^{2}
$$

Eq. (5) is a minimization problem and we will acquire the minimization result by training the network for $\mathrm{m}$ number of weights, here the training will be performed by simulated annealing. It is defined as a probabilistic technique use to find the 
global minimum of constrained and bounded constraint problems that can mathematically reflect the cooling of atoms to a state of lowest energy. A new point is arbitrarily generated at every iteration. The criterion of finding the new point from the current point is based on a probability distribution that depends on the temperature of current state. The procedure takes all new points that lower the objective and with a certain probability it also accepts the point that raise the objective to avoid being trapped in the local minima. The algorithm proceeds by decreasing the temperature till it converges to minimum. Here we used Mathematica 10 to employ the procedure, but the interested readers can read the details of simulated annealing from ref [35]. Training of network will lead us to the final values of network adaptive coefficients that will be replaced in Eq. (4) to get the discretized output of FFIVP at the trial points while the solutions at different points can also be obtained by replacing the trail points array. It is to be noted that the new trail points array will be in the same domain, as was in the training procedure, and should be equally spaced.

\section{NUMERICAL PROBLEM}

EXAMPLE 1. Consider the following linear FFIVP

$D_{t}^{\beta} y(t)-y(t)=0, \quad y(0)=[0.75+0.25 r, 1.125-0.125 r]$

trial solution of the above equation is given as [24]

$\underline{y_{t}}(t)=0.75+0.25 r+t N$

$\overline{y_{t}}(t)=1.125-0.125 r+t N$

While, $N$ can be defined as in Eq. (5) the trial solution in terms of $t$ and $w_{j}$ can be written as, 


$$
\underline{y_{t}}(t)=0.75+0.25 r+\left(\begin{array}{l}
w_{1}+t w_{2}+\frac{1}{2}\left(-1+3 t^{2}\right) w_{3}+\frac{1}{2}\left(-3 t+5 t^{3}\right) w_{4} \\
+\frac{1}{8}\left(3-30 t^{2}+35 t^{4}\right) w_{5}+\frac{1}{8}\left(15 t-70 t^{3}+63 t^{5}\right) w_{6} \\
-\frac{1}{3}\left(\begin{array}{l}
w_{1}+t w_{2}+\frac{1}{2}\left(-1+3 t^{2}\right) w_{3}+\frac{1}{2}\left(-3 t+5 t^{3}\right) w_{4} \\
+\frac{1}{8}\left(\begin{array}{l}
3-30 t^{2} \\
+35 t^{4}
\end{array}\right) w_{5}+\frac{1}{8}\left(15 t-70 t^{3}+63 t^{5}\right) w_{6}
\end{array}\right)^{3} \\
+\frac{2}{5}\left(\begin{array}{l}
w_{1}+t w_{2}+\frac{1}{2}\left(-1+3 t^{2}\right) w_{3}+\frac{1}{2}\left(-3 t+5 t^{3}\right) w_{4} \\
+\frac{1}{8}\left(\begin{array}{l}
3-30 t^{2} \\
+35 t^{4}
\end{array}\right) w_{5}+\frac{1}{8}\left(15 t-70 t^{3}+63 t^{5}\right) w_{6}
\end{array}\right)^{5}
\end{array}\right)^{3}
$$

$$
\bar{y}_{t}(t)=1.125-0.125 r+\left(\begin{array}{l}
w_{1}+t w_{2}+\frac{1}{2}\left(-1+3 t^{2}\right) w_{3}+\frac{1}{2}\left(-3 t+5 t^{3}\right) w_{4} \\
+\frac{1}{8}\left(3-30 t^{2}+35 t^{4}\right) w_{5}+\frac{1}{8}\left(15 t-70 t^{3}+63 t^{5}\right) w_{6} \\
-\frac{1}{3}\left(\begin{array}{l}
w_{1}+t w_{2}+\frac{1}{2}\left(-1+3 t^{2}\right) w_{3}+\frac{1}{2}\left(-3 t+5 t^{3}\right) w_{4} \\
+\frac{1}{8}\left(\begin{array}{l}
3-30 t^{2} \\
+35 t^{4}
\end{array}\right) w_{5}+\frac{1}{8}\left(15 t-70 t^{3}+63 t^{5}\right) w_{6}
\end{array}\right)^{3} \\
+\frac{2}{5}\left(\begin{array}{c}
w_{1}+t w_{2}+\frac{1}{2}\left(-1+3 t^{2}\right) w_{3}+\frac{1}{2}\left(-3 t+5 t^{3}\right) w_{4} \\
+\frac{1}{8}\left(\begin{array}{l}
3-30 t^{2} \\
+35 t^{4}
\end{array}\right) w_{5}+\frac{1}{8}\left(15 t-70 t^{3}+63 t^{5}\right) w_{6}
\end{array}\right)^{5}
\end{array}\right)^{5}
$$

The fractional derivative in the Caputo sense of above trial solutions can be expressed as

$$
\begin{aligned}
\nabla^{\beta} \bar{y}_{t}(t)=\nabla^{\beta} \underline{y_{t}}(t)= & \frac{\Gamma(2)}{\Gamma(2-\beta)} t^{1-\beta}\left(w_{1}-\frac{w_{1}^{3}}{3}+\frac{2 w_{1}^{5}}{15}-\frac{w_{3}}{2}+\ldots\right)+\frac{3}{2} \frac{\Gamma(3)}{\Gamma(3-\beta)} t^{2-\beta} \\
& \left(w_{1}-w_{1}^{2} w_{2}+\frac{2}{3} w_{1}^{4} w_{2} \ldots\right)+\frac{5}{2} \frac{\Gamma(4)}{\Gamma(4-\beta)} t^{3-\beta}\left(-w_{1} w_{2}^{2}+\frac{4}{3} w_{1}^{3} w_{2}^{2}+\ldots\right)+\ldots
\end{aligned}
$$


The MSE equation for $\underline{y}(t)$ and $\bar{y}(t)$ is given as

$$
\begin{aligned}
& M S E=\sum_{i=1}^{10} \frac{1}{10}\left(\nabla^{\beta} \underline{y_{t}}\left(t_{i}, w_{j}\right)-\underline{y_{t}}\left(t_{i}, w_{j}\right)\right)^{2} \\
& M S E=\sum_{i=1}^{10} \frac{1}{10}\left(\nabla^{\beta} \overline{y_{t}}\left(t_{i}, w_{j}\right)-\overline{y_{t}}\left(t_{i}, w_{j}\right)\right)^{2}
\end{aligned}
$$

Training of the network will be done by the process of simulated annealing which we have performed here for specific values of $r$ and $\beta$. Table 1 shows the values of $y(t)$ at $\beta=1, t=1$ and 0.1 at different values of $r$ and the obtained numerical values are compared with RK method, while table 2 shows the numerical comparison at $\beta=$ $0.5, t=0.1$ and at different values of $r$ with modified homotopy perturbation method [18]. Table 3 shows the numerical comparison at $\beta=0.75, t=0.1$ for different values of $r$ and the obtained results are compared with MHPM in [18].

Table 1. Numerical comparison with exact at $\beta=1$

\begin{tabular}{|c|c|c|c|c|c|c|c|c|}
\hline \multirow{3}{*}{$r$} & \multicolumn{5}{|c|}{$t=0.1$} & \multicolumn{4}{c|}{$t=1$} \\
\cline { 2 - 9 } & \multicolumn{2}{|c|}{ LSANN } & \multicolumn{2}{c|}{ Exact } & \multicolumn{2}{c|}{ LSANN } & \multicolumn{2}{c|}{ Exact } \\
\cline { 2 - 9 } & $\underline{y_{t}}(t)$ & $\overline{y_{t}}(t)$ & $\underline{y_{t}}(t)$ & $\overline{y_{t}}(t)$ & $\underline{y_{t}}(t)$ & $\overline{y_{t}}(t)$ & $\underline{y_{t}}(t)$ & $\overline{y_{t}}(t)$ \\
\hline 0 & 0.82832 & 1.24332 & 0.82888 & 1.24332 & 2.03893 & 3.05807 & 2.03871 & 3.05807 \\
\hline 0.1 & 0.84477 & 1.23019 & 0.85651 & 1.22950 & 2.06068 & 3.02380 & 2.10667 & 3.02409 \\
\hline 0.2 & 0.88363 & 1.21568 & 0.88414 & 1.21569 & 2.17472 & 2.99010 & 2.17463 & 2.99011 \\
\hline 0.3 & 0.88121 & 1.20252 & 0.91177 & 1.20187 & 2.13708 & 2.95583 & 2.24258 & 2.95613 \\
\hline 0.4 & 0.93961 & 1.16462 & 0.93940 & 1.18806 & 2.31015 & 2.53515 & 2.31054 & 2.92215 \\
\hline 0.5 & 0.96730 & 1.17486 & 0.96703 & 1.17424 & 2.37811 & 2.88787 & 2.3785 & 2.88817 \\
\hline 0.6 & 0.99499 & 1.14499 & 0.99465 & 1.16043 & 2.44607 & 2.59607 & 2.44645 & 2.85420 \\
\hline 0.7 & 1.02267 & 1.14719 & 1.02228 & 1.14661 & 2.51404 & 2.81990 & 2.51441 & 2.82022 \\
\hline 0.8 & 1.05038 & 1.13335 & 1.04991 & 1.13280 & 2.58309 & 2.78591 & 2.58237 & 2.78624 \\
\hline 0.9 & 1.07801 & 1.11899 & 1.07754 & 1.11899 & 2.64998 & 2.75226 & 2.65032 & 2.75226 \\
\hline 1 & 1.10568 & 1.10568 & 1.10517 & 1.10517 & 2.71795 & 2.71795 & 2.71828 & 2.71828 \\
\hline
\end{tabular}


Table 2. Numerical values at $\beta=0.5$ and $t=0.1$

\begin{tabular}{|l|l|l|l|l|}
\hline & \multicolumn{2}{|l|}{ LSANN } & \multicolumn{2}{l|}{ MHPM } \\
\hline$r$ & $\underline{y_{t}}(t)$ & $\overline{y_{t}}(t)$ & $\underline{y_{t}}(t)$ & $\overline{y_{t}}(t)$ \\
\hline 0 & 1.06143 & 1.59103 & 1.09650 & 1.64475 \\
\hline 0.2 & 1.13293 & 1.45770 & 1.16960 & 1.60820 \\
\hline 0.4 & 1.20364 & 1.42509 & 1.24270 & 1.57165 \\
\hline 0.6 & 1.27325 & 1.48510 & 1.31580 & 1.53510 \\
\hline 0.8 & 1.34387 & 1.44979 & 1.38890 & 1.49855 \\
\hline 1 & 1.32728 & 1.32728 & 1.46200 & 1.46200 \\
\hline
\end{tabular}

Table 3. Numerical values at $\beta=0.75$ and $t=0.1$

\begin{tabular}{|l|l|l|l|l|}
\hline$r$ & \multicolumn{2}{|l|}{ LSANN } & \multicolumn{2}{l|}{ MHPM } \\
\cline { 2 - 5 } & $\underline{y_{t}}(t)$ & $\overline{y_{t}}(t)$ & $\underline{y_{t}}(t)$ & $\overline{y_{t}}(t)$ \\
\hline 0 & 0.898452 & 1.34604 & 0.91369 & 1.37054 \\
\hline 0.2 & 0.958374 & 1.36539 & 0.97460 & 1.34008 \\
\hline 0.4 & 1.01726 & 1.28797 & 1.03552 & 1.30963 \\
\hline 0.6 & 1.07822 & 1.30189 & 1.09643 & 1.27917 \\
\hline 0.8 & 1.09965 & 1.22648 & 1.15734 & 1.24871 \\
\hline 1 & 1.19659 & 1.19659 & 1.21826 & 1.21826 \\
\hline
\end{tabular}

EXAMPLE 2. Consider the nonlinear FFIVP

$D_{t}^{\beta} y(t)+y(t)-y(t)^{2}=0, y(0)=[0.75+0.25 r, 1.5-0.5 r]$

LSANN is utilized on the above nonlinear fractional differential equation by the procedure explained in Example 1 and the acquired results are shown in table 4 for $\beta=1$ and $t=1$ at different values of $r$ and the results in table 5 are for $\beta=0.75$ and $r=0.9$ at different values of $t$ that are compared with the min-max method [19]. 
Table 4. Numerical comparison with exact at $\beta=1$ and $t=1$

\begin{tabular}{|l|l|l|l|l|}
\hline \multirow{2}{*}{$r$} & \multicolumn{3}{|l|}{ LSANN } & \multicolumn{2}{l|}{ Exact } \\
\cline { 2 - 5 } & $\underline{y_{t}}(t)$ & $\overline{y_{t}}(t)$ & $\underline{y_{t}}(t)$ & $\overline{y_{t}}(t)$ \\
\hline 0.5 & 0.720354 & 2.20872 & 0.720292 & 2.19133 \\
\hline 0.6 & 0.768127 & 1.83193 & 0.768031 & 1.82831 \\
\hline 0.7 & 0.819537 & 1.54974 & 0.819403 & 1.54933 \\
\hline 0.8 & 0.875017 & 1.32817 & 0.874839 & 1.32823 \\
\hline 0.9 & 0.935068 & 1.14827 & 0.934842 & 1.14869 \\
\hline 1 & 0.999996 & 0.99999 & 1.000000 & 1.00000 \\
\hline
\end{tabular}

Table 5. Numerical values at $\beta=0.75$ and $r=0.9$

\begin{tabular}{|l|l|l|l|l|}
\hline \multirow{2}{*}{$t$} & \multicolumn{2}{|l|}{ LSANN } & \multicolumn{2}{l|}{ Euler's Method } \\
\cline { 2 - 5 } & $\underline{y_{t}}(t)$ & $\overline{y_{t}}(t)$ & $\underline{y_{t}}(t)$ & $\overline{y_{t}}(t)$ \\
\hline 0 & 0.97500 & 1.05000 & 0.975000 & 1.05000 \\
\hline 0.1 & 0.97041 & 1.06032 & 0.969736 & 1.06154 \\
\hline 0.2 & 0.965865 & 1.07059 & 0.963405 & 1.07595 \\
\hline 0.3 & 0.961363 & 1.08124 & 0.955812 & 1.09403 \\
\hline 0.4 & 0.956705 & 1.09282 & 0.946732 & 1.11689 \\
\hline 0.5 & 0.951698 & 1.10579 & 0.935914 & 1.14607 \\
\hline 0.6 & 0.946256 & 1.12050 & 0.923082 & 1.18373 \\
\hline 0.7 & 0.940407 & 1.13721 & 0.907938 & 1.23310 \\
\hline 0.8 & 0.934183 & 1.15627 & 0.890175 & 1.29913 \\
\hline 0.9 & 0.927427 & 1.17833 & 0.869488 & 1.38979 \\
\hline 1 & 0.919491 & 1.20477 & 0.845595 & 1.51888 \\
\hline
\end{tabular}

\section{CONCLUSION}

In this study, LSANN is effectively established for FIVP and consumed to find the solutions of linear and nonlinear examples of FIVP to exhibit the strength and applicability of the proposed method. The calculations have been done by using the computational software Mathematica 10. Following conclusions can be drawn from the above working.

- Due to the single layer of LSANN, it has lower computational complication and simple implementation compared to multilayer neural network. 
- The results can be obtained over the entire finite domain of integration.

- The thermal minimization methodology made it possible to get the global minima of MSE.

- The LSANN network can handle linear and nonlinear fuzzy fractional differential equations.

- The main advantage of the method is a prediction of accuracy by observing the MSE.

\section{REFERENCES}

[1] Z. Li, D. Chen, J. Zhu, and Y. Liu, Nonlinear dynamics of fractional order Duffing system, Chaos, Solitons \& Fractals, 81 (2015) 111-116.

[2] S. Pourdehi, A. Azami, and F. Shabaninia, Fuzzy Kalman-type filter for interval fractional-order systems with finite-step auto-correlated process noises, Neurocomputing, 159 (2015) 44-49.

[3] A. Boulkroune, A. Bouzeriba, and T. Bouden, Fuzzy generalized projective synchronization of incommensurate fractional-order chaotic systems, Neurocomputing, 173(3) (2016) 606-614.

[4] Y. Ji, L. Su, and J. Qiu, Design of fuzzy output feedback stabilization for uncertain fractional-order systems, Neurocomputing, 173(3) (2016) 1683-1693.

[5] Y. Zheng, Fuzzy prediction-based feedback control of fractional order chaotic systems, Opt. - Int. J. Light Electron Opt., 126(24) (2015) 5645-5649.

[6] S. T. S. Chakraverty, A New Approach to Fuzzy Initial Value Problem by Improved Euler Method, Fuzzy information and Engineering, 4(3) (2012) 293-312.

[7] V. H. Ngo, Fuzzy fractional functional integral and differential equations, Fuzzy Sets Syst., 280 (2015) 58-90.

[8] N. Van Hoa, Fuzzy fractional functional differential equations under Caputo gH-differentiability, Commun. Nonlinear Sci. Numer. Simul., 22 (2015) 1134-1157.

[9] T. Senthilkumar, Robust stabilization and $\mathrm{H} \infty$ control for nonlinear stochastic $\mathrm{T}-\mathrm{S}$ fuzzy Markovian jump systems with mixed time-varying delays and linear fractional uncertainties, Neurocomputing, 173(3) (2016) 1615-1624.

[10] H. Delavari, R. Ghaderi, A. Ranjbar, and S. Momani, Fuzzy fractional order sliding mode controller for nonlinear systems, Commun. Nonlinear Sci. Numer. Simul., 15(4) (2010) 963-978.

[11] S. Salahshour, T. Allahviranloo, and S. Abbasbandy, Solving fuzzy fractional differential equations by fuzzy Laplace transforms, Commun. Nonlinear Sci. Numer. Simul., 17(3) (2012) $1372-1381$.

[12] S. Tapaswini, S. Chakraverty, and D. Behera, Numerical solution of the imprecisely defined inverse heat conduction problem, Chin. Phys. B, 24(5) (2015) 050203

[13] A. Ahmadian, M. Suleiman, and S. Salahshour, An Operational Matrix Based on Legendre Polynomials for Solving Fuzzy Fractional-Order Differential Equations, Abstract and appl. analysis, 2013 Article ID 505903 
[14] T. Allahviranloo', N. Ahmady, and E. Ahmady, Numerical solution of fuzzy differential equations by predictor-corrector method, Information Sciences, 177(7) (2007) 1633-1647.

[15] M.Z. Ahmad, M.K. Hasan, and B.D. Baets, Analytical and numerical solutions of fuzzy differential equations , Information Sciences, 236 (2013) 156-167.

[16] A. Khastan, and K. Ivaz, Numerical solution of fuzzy differential equations by Nyström method, Chaos, Solitons \& Fractals, 41(2) (2009) 859-868.

[17] E. ElJaoui, S. Melliani, and L .S. Chadli, Solving second-order fuzzy differential equations by the fuzzy Laplace transform method, Advances in diff. Eqn., (2015) 2015: 66

[18] N. A. Khan, F.Riaz, and O. A. Razzaq, A comparison between numerical methods for solving fuzzy fractional differential equations, Non Linear Engineering, 3(3) (2014) 155-162.

[19] N. A. Khan, , O. A. Razzaq, and F.Riaz, Numerical Simulations for Solving Fuzzy Fractional Differential Equations by Max-Min Improved Euler Methods, JACSM, 7(1) (2015) 53-83.

[20] H. Sadoghi, M. Pakdaman, and H. Modaghegh, Unsupervised kernel least mean square algorithm for solving ordinary differential equations, Neurocomputing, 74(12-13) (2011) 2062-2071.

[21] S. Mall and S. Chakraverty, Numerical solution of nonlinear singular initial value problems of Emden - Fowler type using Chebyshev Neural Network method, Neurocomputing, 149 (2015) 975-982.

[22] A. J. Meade, The Numerical Solution of Linear Ordinary Differential Equations by Feedforward Neural Networks, 19(12) (1994) 1-25.

[23] A. J. Meade and A. A. Fernandez, Solution of Nonlinear Ordinary Differential Equations by Feedforward Neural Networks, Math.Comp.Model., 20(9) (1994) 19-44.

[24] I. E. Lagaris, A. C. Likas, and D. I. Fotiadis, Artifical Neural Networks for Solving Ordinary and Partial Differential Equations, Neural Networks, IEEE Trans., 9(5) (1998) 1-26.

[25] I. E. Lagaris, A. C. Likas, and D. G. Papageorgiou, Neural-Network Methods for Boundary Value Problems with Irregular Boundaries, 11(5) (2000) 1041-1049.

[26] D. R. Parisi, C. Mariani, and M. A. Laborde, Solving differential equations with unsupervised neural networks, Chem. Eng. Process. Process Intensif., 42 (2003) 715-721.

[27] A. Malek and R. S. Beidokhti, Numerical solution for high order differential equations using a hybrid neural network - Optimization method, Appl. Math. Comput., 183 (2006) 260-271.

[28] H. J. Zimmermann, Fuzzy set theory and its application, Dordrecht, Kluwer Academi Publishers, 1991.

[29] D. Dubois, and H. Prade, Fundamentals of Fuzzy Sets, Kluwer Academic Publishers, USA, 2000.

[30] B.R. Sontakke and A.S. Shaikh, Properties of Caputo operator and its applications to linear fractional differential equations, International Journal of Engineering Research and Applications, 5(5) (2015) 22-29.

[31] M. Caputo, Linear models of dissipation whose Q is almost frequency independent-II, Geophysical Journal International, 13(5) (1967) 529-568

[32] S. Salahshour, T. Allahviranloo, and S. Abbasbandy, Solving fuzzy fractional differential equations by fuzzy Laplace transforms, Commun Nonlinear Sci Numer Simulat, 17 (2012) 1372 1381.

[33] G. J. Klir, and B. Yuan, FUZZY SETS AND FUZZY LOGIC Theory and Applications, Prentice Hall P T R Upper Saddle River, New Jersey 07458 
[34] D. Dubois, and H. Prade, Operations on fuzzy numbers, INT. J. SYSTEMS SCI., 9(6) (1978) $613-$ 626.

[35] S. Ledesma, G.Aviña, and R.Sanchez, Practical considerations for simulated annealing implementation, In: Tan CM, editor. Simulated Annealing, InTech, 2008.

\author{
Najeeb Alam Khan \\ Department of Mathematics, \\ University of Karachi, Karachi 75270, Pakistan \\ email: najeeb_ku@yahoo.com
}

Amber Shaikh

Department of Mathematics, of Karachi, Karachi 75270, Pakistan

Muhammad Asif Zahoor Raja

Department of Electrical Engineering,

COMSATS Institute of Information Technology,

Attock, Pakistan

Sidra Khan

Department of Mathematics,

of Karachi, Karachi 75270, Pakistan 